Historic, Archive Document

Do not assume content reflects current scientific knowledge, policies, or practices. 


$$
\text { . }
$$




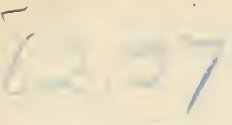

\section{Wholesale Trade List}

Spring 1927

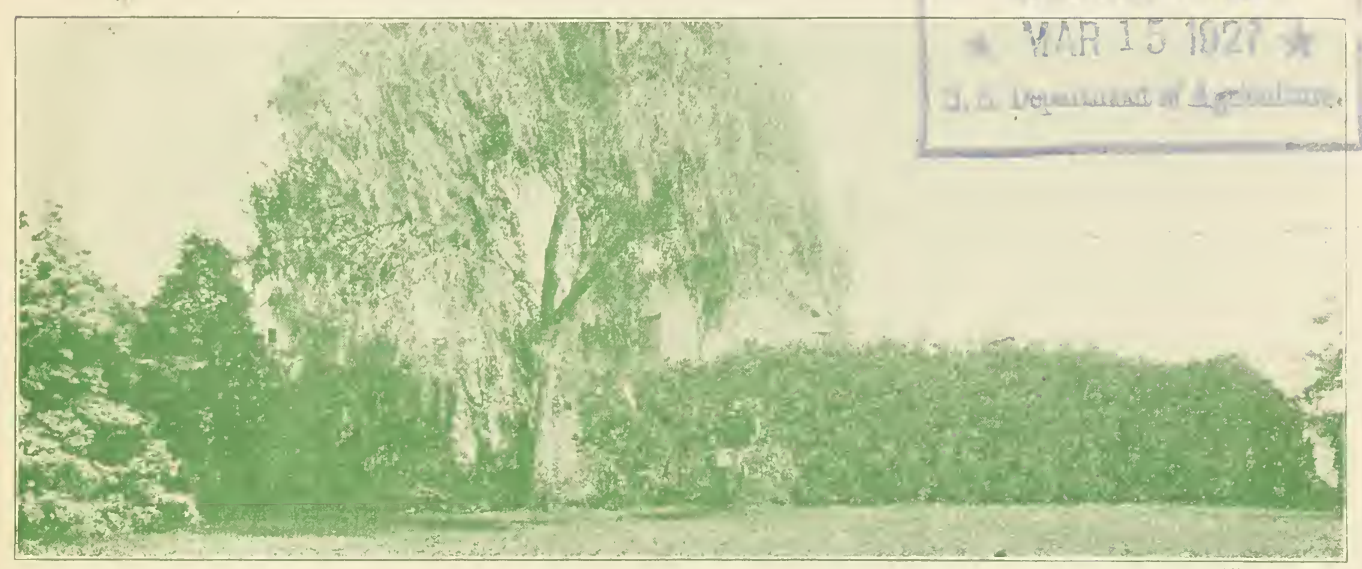

Entrance to Grounds of Evergreen Nursery Co.

\section{EVERGREEN NURSERY CO.}

STURGEON BAY, WIS.

Established $186 \pm$

TERMS AND CONDITIONS

SHIPPING-Please state whether you want goods shipped by freight or express, and also the route, if you have a preference. In the absence of shipping insructions, we will ship according to our discretion, without assuming responsibillty.

PRICES-This list supersedes all previous lists and quotations. Prices quoted are net, and F. O. B. cars here; boxing or baling at cost.

TERIMS-Usual trade terms to those with established credit. Cash or satisfactory trade reference from parties unknown to us.

RATES-Five of any one variety and size sold at ten rates, fifty at one hundred rates, and five hundred at one.thousand rates.

BOXING or BALING will be charged for at actual cost.

CLAIMS for any cause, in order to receive consideration, must be made within six days after receipt of stock.

OUR RESPONSIBILITY for delivery ceases when delivery is made by us to transportation company. All goods travel at risk and expense of purchaser. All orders are accepted subject to loss or injury of stock from fire, drought, or any other causes over which we have no control.

GUARANTY-Every care is experienced to keep our varieties true to name, and we will, when sufficient proof is furnished, replace any which prove otherwise, or else refund the original amount paid for those which proved untrue. It is mutually agreed, however, that we shall in no case be responsible for more than the original purchase price.

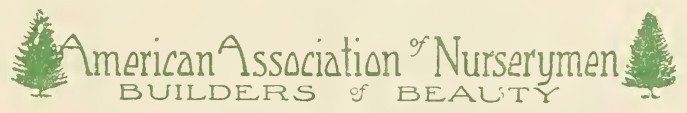




\section{ONCE TRANSPLANTED EVERGREENS}

Our transplants are hard to beat. Climatic and soil conditions in our section of Wisconsin are very conducive to the production of hardy plants with a vigorous root system.

Thuya Occidentalis (Am. Arbor Vitae) $18-24$ inch $12-18$ inch $12-15$ inch $8-12$ inch $6-10$ inch

lbies Balsamea

Ahies Conocolor

Pseudotsuga Douglasi

P'inus I ustriaca 4 - 6 inch $6-10$ inch 4 - 6 inch (Concolor Fir) $6-8$ inch 3 - 6 inch (Douglas Fir) 6 -10 inch ian Pine) $6-10$ inch $4-6$ inch

linus Sylvestris (Scotch Pine) $4-6$ inch

Pinus Strobus

(White Pine) $4-6$ inch

Picea Pungens (Col. Blue Spruce) $6-8$ inch $4-6$ inch

P'icea Excelsa (Norway Spruce) $6-8$ inch

Tsuga Canadensis

(Hemlock) 6-10 incli
100

$\$ 18.00$

12.00

10.00

7.50

6.50

12.00

7.00

20.00

15.00

11.00

9.00

8.00

6.50

5.50

6.00

1000

$\$ 150.00$

110.00

95.00

70.00

(;). ()1)

22.00

15.00

6.00

50.08

15.00
100.00 80.00

(6). 00

50.00

50.00

\section{'TWICE. TRA YSPLINTEI)}

We pride ourselves on our nice lot of twice transplanted stock. They are ideal for lining out for future specimen stock.

Thuya 0ccidentalis (Am. Arbor Vitae)

$\$ 6.00$

(Austrian Pine)

$10-12$ inch

(Scotch Pine)

$18-24$ inch

$12-18$ inch

Picea Canadensis (Black Hills Spruce)

8-12 inch

Picea Alha

Picea Excelsa

tbies Balsamea

Tsuga Canadensis
(White Spruce)

18-24 inch 12-18 inch

(Norway Spruce)

18-24 inch

12-18 inch

8-12 inch

(Balsam Fir)

12-15 incl

8-12 inch

(Hemlock)

12-15 inch
10

100

5.00

$\$ 50.00$

45.00

5.00

3.50

45.00

30.00

4.00

35.00

6.00

50.00

4.00

35.00

2.50

22.00

2.00

18.00

1.75

15.00

8.00

75.00

6.00

50.00

5.50

50.00 


\section{EVIRGEEI SELULIVGS}

Let us call your attention especially to our Am. Arbor Vitae seedlings. which has been our big seller in seedlings as well as in transplants. They must be good.

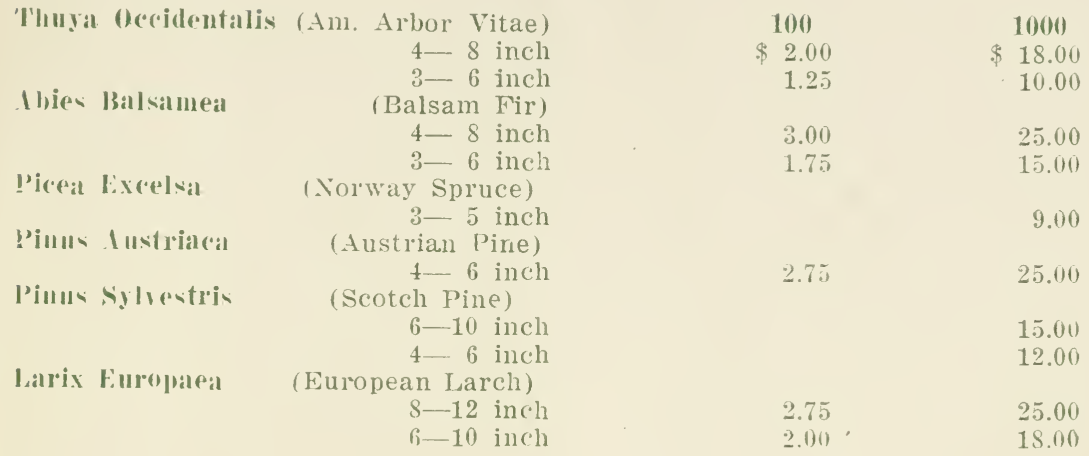

\section{FORES'T 'TREE SEEDLIYGS}

Our maple seedlings are not mere whips, but are good strong plants. iceal for lining out.

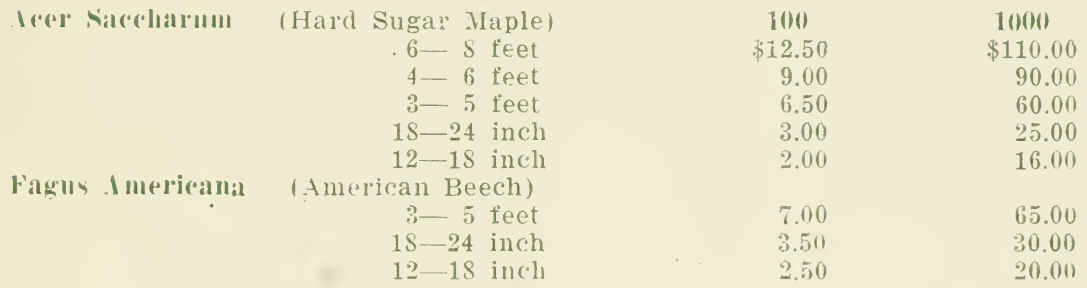

The following one year seedlings and once tpd. stock will be shipped direct from our plant in eastern Kansas.

\section{ONE YEIR SEEIOLIYS}

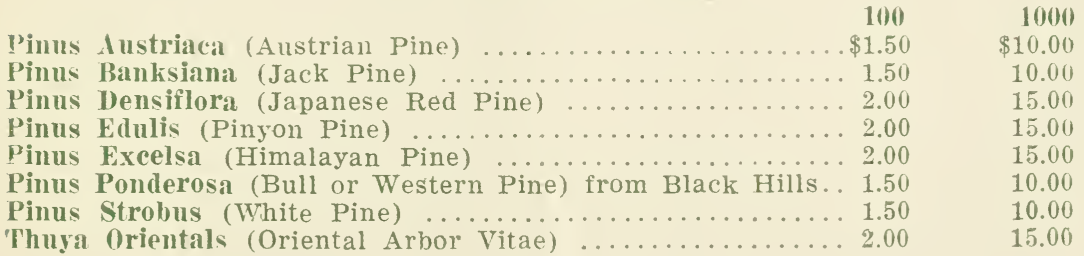

\section{ONCE TRANSPLAN'TEI)}

$\begin{array}{lr}4-6 \text { inch } & 12.00 \\ 6-8 \text { inch } & 15.00\end{array}$




\section{SPECIMEN EVERGREENS}

This stock is several times transplanted, very well rooted, and symmetrical in every respect. Prices include B \& B.

Picea Alba

P'ieea Excelsa

l'inus Austriaca

Juniperu Sabina (White Spruce)

$$
\begin{array}{lll}
11 / 2-2 & \text { feet } \\
1 & -11 / 2 & \text { feet } \\
8 & -12 & \text { inch }
\end{array}
$$

(Norway Spruce)

$$
\begin{array}{lll}
3 & -4 & \text { feet } \\
2 & -3 & \text { feet } \\
1 & -11 / 2 & \text { feet }
\end{array}
$$

(Austrian Pine)

$$
\begin{array}{lll}
2 & -3 & \text { feet } \\
10 & -12 & \text { inch }
\end{array}
$$$$
\text { (Savin Juniper) }
$$

$$
1-1 \frac{1 / 2}{\text { feet }}
$$

Thuya 0ccidentalis (Am. Arbor Vitae)

$$
1 \frac{1}{2}-2 \text { feet }
$$$$
1-1 \frac{1}{2} \text { feet }
$$

Thuya Occidentalis Hovey (Hovey Arbor Vitae)

$$
1-1 \frac{1}{2} \text { feet }
$$

Thuya Occidentalis Globosa (Globe Arbor Vitae)

$$
1-1 \frac{1 / 4}{4} \text { feet }
$$

Whuya 0ccidentalis Wareana (Siberian A. V.)

Thuya Occidentalis Erecta

$$
1-1 \frac{1 / 4}{4} \text { feet }
$$

$$
1-1 \frac{1 / 2}{2} \text { feet }
$$

Thuya Occidentalis Pyramidalis (Pyramidal A. V.)

\section{Retinospora Plumosa}

$1-1 \frac{1}{2}$ feet

$11 / 2-2$ feet

'T'suga Canadensis

$1-1 \frac{1 / 2}{\text { feet }}$

(Hemlock)

12 inches each

$\$ 1.00$

.75

.50

1.75

1.50

.50

1.25

.75

1.50

1.00

.75

1.00

1.00

1.25

1.50

1.25

1.75

2.00

.75
10)

$\$ 9.00$

7.00

4.50

16.00

14.00

4.00

12.00

7.00

14.00

9.00

7.00

9.50

9.50

10.00

12.00

11.00

16.00

19.00

7.00 